\title{
PREOPERATIVE INVESTIGATIONS IN ELECTIVE SURGERY: PRACTICES AND COSTS AT THE NATIONAL HOSPITAL OF SRI LANKA
}

\author{
P. Ranasinghe ${ }^{1}$, .Y.S. Perera ${ }^{2}$, A. Abayadeera ${ }^{3}$ \\ Research Associate, Diabetes Research Unit, Department of Clincal Medicine, Faculty of Medicine, \\ University of Colombo, Sri Lanka ${ }^{l}$, Research Assistant, Department of Clincal Medicine, Faculty of \\ Medicine, University of Colombo, Sri Lanka ${ }^{2}$, Senior Lecturer, Department of Surgery, Faculty of \\ Medicine, University of Colombo, Sri Lanka ${ }^{3}$
}

*Corresponding author: rana90210@yahoo.com

Key words: Anaesthesia, audit; Surgery, preoperative period; Assessment, preanaesthetic

\begin{abstract}
Background:
During preoperative preparation patients undergo investigations to detect asymptomatic diseases. The probability of finding significant abnormalities on such "routine" investigations is small, unnecessarily increasing costs of perioperative care. We evaluated current practices, compliance with guidelines and costs of preoperative investigations at the National Hospital of Sri Lanka (NHSL).
\end{abstract}

\begin{abstract}
Methods:
Patients undergoing elective surgery at seven general surgical units of the NHSL from JanuaryFebruaary 2009 were included. NICE-UK guidelines on pre-operative investigation was the standard of assessment. Data on preoperative investigations were collected using an expert validated pre-tested interviewer administered questionnaire. A self-administered questionnaire was used to assess the ward practices and House Officers'(HOs) knowledge.
\end{abstract}

\section{Results:}

Sample size was 367 . Mean age $45.7 \pm 15.8$ years and males were $46.9 \%$. Majority were ASA-I $(68.7 \%)$ and surgical grade-II (56.7\%). Urinalysis and arterial blood gases demonstrated good adherence $(70 \%-100 \%)$ to guidelines. Electrocardiography, full blood count, renal function tests and blood grouping demonstrated moderate adherence (40-69\%), all other investigations demonstrated poor adherence $(<40 \%)$. Non-recommended investigations were requested mostly by the HOs and Medical Officers and registrars in anaesthesia. Total excess cost incurred due to non-recommended investigations during the study period was LKR 241,300 375,270 .

HOs decisions on preoperative investigations were based on; past experience of similar patients, directives given by seniors or their clinical training. Investigations requested by all members of the surgical/anesthetist team are commonly done with minimal utilization of guidelines. The HOs ability to plan preoperative assessment for model case scenarios was insufficient.

\section{Conclusions:}

Unnecessary preoperative investigations are common at our institution leading to substantial excess costs. There is ample opportunity to rationalize practices and reduce expenditure.

Patients admitted to hospital for elective surgery commonly undergo a battery of "routine" preoperative investigations, often including a complete blood count, renal function tests, blood glucose level, urinalysis, chest X-ray (CXR) and an electrocardiogram (ECG). The goal of such investigations is to detect asymptomatic diseases not apparent on history and examination. If 
identified prior to surgery they could alter anaesthetic/surgical management. Appropriate treatment of these diseases helps to minimize perioperative morbidity and mortality. ${ }^{1}$

However in the absence of any clinical indication, the probability of finding a significant abnormality on laboratory tests ${ }^{2,3}$, ECGs and CXRs ${ }^{4,5}$ are small. Even among elderly patients "routine" preoperative testing was of little benefit. ${ }^{6}$ A randomized controlled trial of preoperative testing in cataract surgery revealed no differences in outcome between those subjected to and not subjected to routine testing. ${ }^{7}$

Routine use of large number of screening tests increases the costs of perioperative care. ${ }^{8}$ Unnecessary testing may cause harm to the patient due to over treatment for borderline/false-positive results. Hence the indiscriminate use of such investigations remains a matter for discussion, since costs may be increased without reducing perioperative complications. ${ }^{9}$

Various agencies including National Institute of Health and Clinical Excellence(NICE-UK) ${ }^{10}$ and Canadian Anesthesiologists' Society(CAS) ${ }^{11}$ have published guidelines to rationalize testing practice and reduce costs. To our knowledge there are no published studies evaluating preoperative testing practices and costs involved in Sri Lanka.

The primary objective of this study was to understand current practices of preoperative testing and determine if they were compliant with the NICE-UK guidelines on preoperative testing. Secondary objectives included: identification of the source of testing orders non-compliant with guidelines, evaluating factors influencing decisions and evaluation of excess costs incurred due to unnecessary preoperative investigation.

\section{Methods}

\section{Study population}

The study was conducted at the NHSL, a tertiary care unit with 3000 beds. Each surgical team comprises of a Consultant Surgeon, a Senior Registrar (SR), Registrars, Senior House Officers (SHOs) and House Officers (HOs). In addition a consultant anaesthesiologist is in charge of preoperative assessment at each unit under whom either a Medical Officer - Anaesthesia (MO anaesthesia) or a Registrar-Anaesthesia visits the respective ward for preoperative assessment. This prospective study was undertaken among all patients undergoing elective surgery at seven general surgical units of the NHSL for a period of one month from January-February 2009. Patients undergoing emergency surgery were excluded.

NICE-UK guidelines on pre-operative investigation was the standard of assessment, developed by NICE in conjunction with the National Collaborating Centre for Acute Care in UK. Details of the evidence, method and the guidance can be found on their website. ${ }^{10}$ The guidelines take into account the complexity of the surgery, American Society of Anesthesiologists (ASA) status of the patient and the major comorbidity. For each test, a colour coded system highlights if test is indicated or not. These guidelines tailor the patient to the procedure using as much evidence as possible, while allowing sufficient leeway for clinical judgment.

The following investigations were evaluated; Chest X-Ray (CXR), Electrocardiogram (ECG), Full blood count (FBC), Prothrombin time/International normalized ratio (PT/INR), Fasting/Random blood sugar (FBS/RBS), Urine analysis, Arterial blood gas (ABG), Renal function tests (RFT), Lung function tests (LFT), AST/ALT, Blood grouping \& cross-matching and 2D Echocardiogram (2D-Echo). The recommendations for AST/ALT, blood grouping $\&$ cross-matching and 2D-Echo was obtained after an iterative consensus process among a panel of experts. Remaining investigations were evaluated according to NICE-UK guidelines. The price of each investigation was obtained from ten government and non-government institutions.

NICE-UK guidelines recommend that urine dipstick analysis to be considered in all patients undergoing elective surgery, which is not routinely done in Sri Lanka due to financial constraints. However all patients on admission have a 'urine ward-test' to examine urine for glucose, protein and etc. This was taken as comparable to urine dipstick analysis.

\section{Data Collection}

Data were collected using an expert-validated pretested interviewer administered questionnaire designed following the NICE-UK guidelines and 
ASA classification of patients. Ward practices on preoperative evaluation were assessed using a separate self-administered questionnaire distributed among HOs of the surgical units, it also evaluated the HOs knowledge on preoperative evaluation using ten commonly encountered general surgical case-scenarios developed and assessed using NICE-UK guidelines. The HOs were asked to record the surgical grade, ASA grade and to select appropriate investigations from a list of ten investigations given for each case. Each correct surgical grade, ASA grade and investigation was given one mark. Thus total marks per questionnaire for surgical grades, ASA grades and investigations were 10, 10 and 100 respectively. Ethical approval was obtained from the Ethics Review Committee of NHSL.

\section{Statistical analyses}

Data were double entered and cross checked for consistency. Analysis was done using SPSS v14 (SPSS Inc.,Chicago,IL,USA). Categorical data were described as proportions. Continuous data were described using mean and standard deviation and compared using unpaired Student's t tests. A $\mathrm{p}$ value of $<0.05$ was considered statistically significant.

\section{Results}

During the study period records of 367 patients were studied. Mean age was $45.7 \pm 15.8$ years and males were $46.9 \% \quad(n=172)$. Majority $(68.7 \%, \mathrm{n}=272)$ of patients had no co-morbidities (ASA-I). Most patients underwent intermediate grade (Grade-2) surgical procedures $(n=208 / 56.7 \%)$. Sample characteristics are summarized in table 1.

The mean number of investigations requested per patient was 4.58 ( $\mathrm{SD} \pm 2.00)$. FBC was the most requested investigation $(n=347,95.1 \%)$.

For each investigation compliance/noncompliance to guideline and cost incurred due to non-compliance were evaluated. Request for AST/ALT and PT/INR least conformed to guidelines (Table 2).

Table 1 - Characteristics of the study population

\begin{tabular}{|l|c|c|}
\hline Characteristic & Number of patients & Percentage (\%) \\
\hline Gender & 172 & $46.9 \%$ \\
Male & 195 & $53.1 \%$ \\
Female & 252 & \\
\hline ASA Classification & 109 & $68.7 \%$ \\
Grade I & 6 & $29.7 \%$ \\
Grade II & & $1.6 \%$ \\
Grade III & 252 & \\
\hline Co-morbid illnesses & 59 & $68.7 \%$ \\
No illness/healthy & 40 & $16.1 \%$ \\
Hypertension & 35 & $10.9 \%$ \\
Diabetes mellitus & 30 & $9.5 \%$ \\
Bronchial asthma & 68 & $8.2 \%$ \\
Ischaemic heart disease & 208 & $18.5 \%$ \\
\hline Surgical grading & 72 & $56.7 \%$ \\
Grade 1 & 19 & $19.6 \%$ \\
Grade 2 & & $5.2 \%$ \\
Grade 3 & $16 \%$ \\
Grade 4 & 168 & \\
\hline Surgical procedure & & \\
Inguinal herniotomy & 62 & $16.8 \%$ \\
Thyroidectomy & 50 & $13.6 \%$ \\
Para-umbilical herniotomy & 31 & $8.4 \%$ \\
Varicose vein surgery & 22 & $6.0 \%$ \\
Hydrocelectomy Laparoscopic & 18 & $4.6 \%$ \\
cholecystectomy & & $4.3 \%$ \\
Other & 16 & $45.8 \%$ \\
\hline *41 patients had multiple co-morbid illnesses &
\end{tabular}

*41 patients had multiple co-morbid illnesses 
Table 2 - Investigations done compared with NICE-UK guidelines and costs of nonrecommended investigations

\begin{tabular}{|c|c|c|c|c|}
\hline $\begin{array}{l}\text { Investigation } \\
\text { (no. done) }\end{array}$ & $\begin{array}{l}\text { Recommend } \\
\text { ed }(\%) \S\end{array}$ & $\begin{array}{l}\text { Consider } \\
(\%) \S\end{array}$ & $\begin{array}{l}\text { Not } \\
\text { recommended } \\
(\%) \S\end{array}$ & Price (Rs.) ${ }^{\star}$ \\
\hline LFT (11) & $0(0.0 \%)$ & $0(0.0 \%)$ & $11(100 \%)$ & $9,350-16,500$ \\
\hline AST/ALT (47) & $5(10.6 \%)$ & $0(0.0 \%)$ & $42(89.4 \%)$ & $12,600-22,680$ \\
\hline PT/INR (126) & $1(0.8 \%)$ & $14(11.1 \%)$ & $111(88.1 \%)$ & $33,330-63,270$ \\
\hline 2D Echo (52) & $15(28.8 \%)$ & $1(1.9 \%)$ & $36(69.2 \%)$ & $72,000-82,800$ \\
\hline Blood sugar (255) & $0(0.0 \%)$ & $101(39.6 \%)$ & $154(60.4 \%)$ & $15,400-30,800$ \\
\hline CXR (177) & $0(0.0 \%)$ & $70(39.6 \%)$ & $107(60.4 \%)$ & $32,860-49,820$ \\
\hline RFT (245) & $58(23.7 \%)$ & $91(37.1 \%)$ & $96(39.2 \%)$ & $14,400-26,400$ \\
\hline Blood grouping (108) & $67(62.0 \%)$ & $0(0.0 \%$ & $41(38.0 \%)$ & $7,380-12,710$ \\
\hline FBC (347) & $113(32.6 \%)$ & $125(36.0 \%)$ & $109(31.4 \%)$ & $22,890-35,970$ \\
\hline ECG (310) & $93(30.0 \%)$ & $122(39.4 \%)$ & $95(30.6 \%)$ & $21,120-34,320$ \\
\hline Urine analysis (367) & $0(0.0 \%)$ & $\begin{array}{l}367 \\
(100.0 \%)\end{array}$ & $0(0.0 \%)$ & 0 \\
\hline $\mathrm{ABG}(1)$ & $0(0.0 \%)$ & $1(100 \%)$ & $0(0.0 \%)$ & 0 \\
\hline Total (2046) & $352(17.2 \%)$ & $900(44.0 \%)$ & $794(38.8 \%)$ & $241,300-375,270$ \\
\hline
\end{tabular}

* Price $=$ number of investigations not recommended $\mathrm{x}$ price range of investigation $\S=$ proportion of investigations from total number of investigations done

To calculate cost incurred for each investigation both minimum and maximum prices were used to obtain a cost range as prices of investigations varied between institutions. PT/INR, CXR and 2D-Echo were the investigations which cost most due to non-compliance with guidelines(Table 2). The total excess cost incurred due to nonrecommended investigations during the study period was LKR. 241,300-374,270 (per patient LKR. 666.57-1,033.90). The annual excess expenditure incurred for the seven general surgical units by the NHSL was extrapolated to be LKR. $2,895,600-4,491,240$. Investigations that did not comply with the guidelines were requested in most instances by either the HOs or MOAnaesthesia/Registrar-Anaesthesia(Table 3).

Table 3 - Who requests the investigations non-compliant with the guidelines?

\begin{tabular}{|l|c|c|c|c|c|c|}
\hline $\begin{array}{l}\text { Investigation } \\
\text { (number not } \\
\text { recommended) }\end{array}$ & HO & SHO & Registrar & SR & Consultant & Anesthetist§ \\
\hline CXR (106) & 98 & 1 & 5 & 1 & 3 & 54 \\
\hline ECG (88) & 81 & 0 & 3 & 0 & 4 & 46 \\
\hline FBC (109) & 103 & 0 & 2 & 0 & 10 & 34 \\
\hline PT/INR (111) & 104 & 3 & 8 & 1 & 5 & 35 \\
\hline Blood sugar (154) & 132 & 1 & 10 & 2 & 14 & 58 \\
\hline RFT (96) & 93 & 2 & 1 & 0 & 3 & 22 \\
\hline LFT (11) & 11 & 0 & 0 & 0 & 2 & 9 \\
\hline AST/ALT (42) & 36 & 0 & 4 & 1 & 0 & 14 \\
\hline 2D Echo (36) & 19 & 1 & 3 & 3 & 1 & 22 \\
\hline Blood grouping (41) & 33 & 0 & 4 & 0 & 1 & 11 \\
\hline
\end{tabular}

*Urine analysis and ABG which showed $100 \%$ adherence to guidelines is not included, § MO- 
Anaesthesia or Registrar-AnaesthesiaThe response rate for ward practices and $\mathrm{HO}$ knowledge questionnaire was $83.3 \%$ (20 out of 24 HOs). HOs based their decision on preoperative investigation either by past experience of similar patient, directives given by seniors or their clinical training. Utilization of guidelines was minimal. None of the HOs were aware about any published guideline on preoperative investigations. In most surgical units investigations requested by all members of the surgical/anaesthetist team were done (Table 4)

Table 4 - Ward practices

\begin{tabular}{|l|c|c|c|c|}
\hline & Most of the time & Sometimes & Rarely & Total \\
\hline $\begin{array}{l}\text { My/HOs decision on } \\
\text { preoperative investigations } \\
\text { are based upon }\end{array}$ & & & & \\
Past experience of a similar & $11(50.0 \%)$ & $9(45.0 \%)$ & $0(0.0 \%)$ & $20(100 \%)$ \\
patient & $14(70.0 \%)$ & $6(30.0 \%)$ & $0(0.0 \%)$ & $20(100 \%)$ \\
Directives given by seniors & $17(85.0 \%)$ & $3(15.0 \%)$ & $0(0.0 \%)$ & $20(100 \%)$ \\
My medical training & $0(0.0 \%)$ & $9(45.0 \%)$ & $11(55.0 \%)$ & $20(100 \%)$ \\
A set of guidelines & & & & \\
\hline All investigations are done & $17(85.0 \%)$ & $2(10.0 \%)$ & $1(5.0 \%)$ & $20(100 \%)$ \\
\hline
\end{tabular}

HOs demonstrated insufficient knowledge on the prices of most investigations. The estimated price of investigations given by the HOs had a wider range and significantly different mean from the actual price. The HOs estimated prices were comparable to the actual price only for CXR, FBC, Urine analysis and 2D-Echo (Table 5). The mean score $( \pm \mathrm{SD}$, range) of HOs for the ten model case scenarios were as follows; correct surgical grade $2.85( \pm 3.96,0-8)$, ASA grade $4.65( \pm 3.96,0-10)$ and investigation $47.4( \pm 12.46,0-58)$. The number of investigations done showed a significant positive correlation with patients' age $(r=0.378)$, surgical grade $(r=0.564)$ and ASA grade $(r=0.216)$.

\section{Table 5 - Estimation of prices of investigations by Hos}

\begin{tabular}{|l|l|l|l|}
\hline & Actual price & Estimated price & \multirow{2}{*}{ P Value } \\
\cline { 2 - 4 } & Mean (Range) & Mean (Range) & \\
\hline CXR & $371.67(310-470)$ & $344.12(100-1000)$ & 0.6 \\
\hline ECG & $340.00(240-390)$ & $264.41(50-500)$ & $<0.05$ \\
\hline FBC & $274.38(210-330)$ & $285.29(50-400)$ & 0.6 \\
\hline PT/INR & $475.00(300-570)$ & $657.14(300-1400)$ & $<0.05$ \\
\hline Blood Sugar & $161.88(100-200)$ & $230.00(80-400)$ & $<0.01$ \\
\hline Urine Analysis & $146.25(80-200)$ & $170.00(60-300)$ & 0.1 \\
\hline ABG & $1433.33(1300-1500)$ & $686.36(500-1000)$ & $<0.01$ \\
\hline RFT & $220.63(150-275)$ & $827.57(200-1800)$ & $<0.05$ \\
\hline LFT & $1116.67(850-1500)$ & $1886.36(1000-2500)$ & $<0.01$ \\
\hline AST/ALT & $455.00(300-540)$ & $960.71(300-2000)$ & $<0.05$ \\
\hline 2D Echo & $2212.5(2000-2300)$ & $2103.57(400-5000)$ & 0.6 \\
\hline Blood Grouping & $262.86(180-310)$ & $476.67(180-1000)$ & $<0.05$ \\
\hline
\end{tabular}




\section{Discussion}

Preoperative assessment is a key process in minimizing morbidity of surgery. Diagnostic studies should be used as an adjunct to findings obtained from a review of history and examination or ordered to ensure that clinically silent conditions which can influence perioperative outcome are detected.

Majority of our sample population were healthy patients undergoing intermediate grade surgical procedures. Junior members of the team with less experience were involved in planning investigations in most patients. Involvement of senior members of the team on planning investigations was minimal. When investigations were evaluated for adherence to guidelines, urine analysis and $\mathrm{ABG}$ were the only investigations with good adherence (70\%-100\% compliance). It is mandatory in Sri Lanka that all patients have urine analysis, while $\mathrm{ABG}$ was requested only in one patient during the study period, resulting in these investigations demonstrating a good compliance to guidelines. ECG, FBC, RFT and blood grouping demonstrated moderate adherence (40-69\% compliance). All other investigations demonstrated poor adherence ( $<40 \%$ compliance) to guidelines. A substantial excess cost is incurred by the hospital for unnecessary preoperative testing. This study was limited to the general surgical units thus actual total expenditure incurred due to unnecessary investigations by the hospital could be substantially higher.

When evaluating the source of testing orders noncompliant with guidelines the HOs and MOAnaesthesia/Registrar-Anaesthesia were responsible for nearly all such requests. Previous studies have demonstrated that selective ordering of preoperative investigations by specialist anesthesiologists reduces the number and cost of tests. ${ }^{12}$ Other contributory factors were minimal utilization of guidelines, lack of awareness and performing investigations requested by all members of the team without supervision. In addition the HOs who were responsible for planning preoperative investigations in most patients demonstrated a poor knowledge on planning preoperative investigations for model case scenarios and also showed lack of awareness about the prices of investigations.
The results suggest that unnecessary laboratory testing during preoperative preparation of patients is still common. A substantial excess cost is incurred due to this. There is ample opportunity to rationalize testing practice and decrease testingrelated costs without altering outcome. We recommend the following remedies; (1). Education of surgical and anaesthetic teams on current practices and resulting costs (2). Adoption of guidelines on preoperative investigations aiming to modify existing practices (3). Re-evaluation after adopting guidelines.

\section{Acknowledgements}

We would like to thank the surgical and anaesthetic team members of the general surgical units of the National Hospital of Sri Lanka.

\section{References}

1. Roizen M. Preoperative patient evaluation. Canadian Journal of Anaesthesia 1989; 36(3Pt2) : S13-9.

2. Adams JG jr, Weigelt JA, Poulos E. Usefulness of preoperative laboratory assessment of patients undergoing elective herniorrhaphy. Archives of Surgery 1992; 127: 801-5.

3. Narr BJ, Hansen TR, Warner MA. Preoperative laboratory screening in healthy Mayo patients: cost-effective elimination of test and unchanged outcomes. Mayo Clinic proceedings 1991; 66: 1559.

4. Gagner M, Chiasson A. Preoperative chest X-ray films in elective surgery: a valid screening tool. Canadian Journal of Surgery 1990; 33: 271-4.

5. Tape TG, Mushlin AI. The utility of routine chest radiographs. Annals of Internal medicine 1986; 104: 663-70.

6. Dzankic S, Pastor D, Gonzalez C, Leung JM. The prevalence and predictive value of abnormal preoperative laboratory tests in elderly surgical patients. Anesthesia \& Analgesia 2001; 93: 301-8.

7. Schein OD, Katz J, Bass EB, Tielsch JM, Lubomski LH, et al. The value of routine preoperative medical testing before cataract surgery. Study of Medical Testing for Cataract Surgery. New England Journal of Medicine 2000; 342: 168-75.

8. Relman AS. Assessment and accountability: the third revolution in medical care. New England Journal of Medicine. 1988; 319(18): 1220-2.

9. France FH, Lefebvre C. Cost-effectiveness of preoperative examinations. Acta Clinica Belgica. 1997; 52(5): 275-86. 
10. National Collaborating Centre for Acute Care. CG3 Preoperative tests, the use of routine preoperative tests for elective surgery: evidence, methods and guidance - Full guideline. National Institute for Health and Clinical Excellence. Available from URL; http://www.nice.org.uk/page.aspx?o=77801. (accessed 20/11/2008).

11. Canadian Anesthesiologists' Society. Guidelines to the practice of anesthesia. The pre-anesthetic period.Available from URL; http://www.cas.ca/members/sign_in/guidelines/pra ctice_of_anesthesia/default.asp?load=preanesthetic . 2004 (accessed 20/11/2008).
12. Finegan BA, Rashiq S, McAlister FA, O'Connor P. Selective ordering of preoperative investigations by anesthesiologists reduces the number and cost of tests. Canadian Journal of Anaesthesia 2005; 52: 575-80.

13. number and cost of tests. Canadian Journal of Anaesthesia 2005; 52: 575-80. 